\title{
Development of Event Manager and Its Application in Jini Environment*
}

\author{
YoungLok Lee ${ }^{1}$, HyungHyo Lee ${ }^{2}$, Seungyong Lee ${ }^{1}$, InSu Kim ${ }^{1}$, and BongNam Noh ${ }^{1, * *}$ \\ ${ }^{1}$ Dept. of Information Security, Chonnam National University, Gwangju 500-757, Korea \\ \{dogu, birch, agisong, bongnam\}@lsrc.jnu.ac.kr \\ ${ }^{2}$ Div. of Information and EC, Wonkwang University, Iksan 570-749, Korea \\ hlee@wonkwang.ac.kr
}

\begin{abstract}
Ubiquitous computing services have to adapt to the context information. These services have to communicate with each other through fixed network or ad-hoc, it is the ubiquitous middleware to be able to help those services. With regard to the adaptation of middleware's components, context manager and event manager are required. Recently there is a widely used middleware, Jini, but it is distributed without the event manager services. Therefore we implement the event manages which can manage events in Jini environments, and describe ubiquitous computing applications running environment using our event management system. Our event manager is implemented by modifying Javaspaces.
\end{abstract}

\section{Introduction}

An event in ubiquitous computing can be defined as an object that contains information about external status in which other software components are interested. There are various events ranged from low level signals generated by sensors to deduced valuable information in high level. Users in the ubiquitous computing environment should be able to adapt themselves to their current context information and high level information generated by these events.

Jini, the Home Network middleware, helps new service components connect to Home Networks at any time and helps clients promptly use them without extra settings, and even in the case of service component upgrade, the existing client service can operate with no problems. If there are any interested changes in the outer world, whether Jini services are in local or not, it is necessary for them to be asynchronously notified. Jini can achieve it by using the event notification concepts as other java components. Jini's lookup service finds the service which client is looking for and, if found, hands over a proxy. However if not found, the client registers themselves with the lookup service as a remote event listener so that she can be notified when the event she is looking for is written to lookup.

* This research was supported by the MIC (Ministry of Information and Communication), Korea, under the ITRC (Information Technology Research Center) support program supervised by the IITA (Institute of Information Technology Assessment).

*** Corresponding author.

T. Enokido et al. (Eds.): EUC Workshops 2005, LNCS 3823, pp. 704-713, 2005.

(C) IFIP International Federation for Information Processing 2005 
Even though there is a way of using Javaspace services or lookup services in order to manage events. In the ubiquitous environments which contain a lot of sensors or context managers, we need to systematically manage events by putting distributed event managers. By modifying the JavaSpace, we develop the event manager to manage events.

This paper consists of as follows;

Chapter 2 shows related work, and chapter 3 explains the design and implementation of event management system based on JavaSpace. In chapter 4, we explain how context managers and application services can utilize our event manager. Finally, makes a conclusion and further research works.

\section{Related Work}

The CORBA Event Service[1] standardized the transfer of asynchronous notifications between objects. An object that generates events is called supplier whereas an object which receives events is called consumer. A supplier passes an event to a consumer by invoking an appropriate method on the consumer interface. Suppliers and consumers can be decoupled from each other by event channel objects. An event channel forwards all events it receives from any of its suppliers to all the consumers that have registered with the channel. The events themselves can be either of generic or typed format. The standardized CORBA Notification Service[2] developed by the OMG Telecommunications Domain Task Force provide much more flexible event filtering capabilities. It is possible to establish user-defined event filters by assigning priorities to events, generate timestamps, and introduce QoS criteria for the handling of events.

One of middlewares is the Gaia of the University Illinois under active research. The event manager of Gaia[3] satisfies many of general needs in event management. The event manager distributes load among multiple machines. It creates event channel factories remotely on pre-configured machines whenever it decides that the existing event channel factories are already overloaded. In essence, the event manager keeps state for the channels it creates and recreates them if they crash, transparent to the event consumers. Event manager service implementation of Gaia makes use of CORBA event service as the basic event storage.

The Java Message Service (JMS)[4] is a Java API that allows applications to create, send, receive and understand messages. It defines a common set of interfaces and associated semantics that allows Java programs to communicate with other messaging implementations. JMS provides a loosely coupled architecture that supports asynchronous communication and guarantees reliable delivery of messages. The specification provides for both point-to-point messaging using queues and the publisher/subscriber approach using topics as intermediaries. Messages can be consumed both synchronously ("pull") and asynchronously ("push"). It also has message filtering capabilities in the form of message selectors based on a subset of SQL92 conditional expression syntax.

Distributed events in the Jini[5] framework allow an object in one virtual machine (VM) to register interest in the occurrence of an event in another object, possibly running in another VM, and receive notification when such an event happens. The Jini Event System uses the Jini Lookup Service for naming which can be optionally used 
with Java Native Directory Interface (JNDI)[6]. An event is a Java object that can be subtyped for extensibility. The listener interface is simple and aids in the use of a flexible publisher model. Jini supports leasing and uses Java-RMI as the communication substrate. It leverages the built-in security of Java. However, Jini events are designed to work only in the Java environment and are not equipped to work with firewalls and Network Address Translation (NAT).

\section{JavaSpace-Based Event Management System in Jini}

JavaSpaces $^{\mathrm{TM}}$ are a networked repository for java objects. One of the functions of JavaSpace is to notify an event to the entity who is interested in the event object when that event object is stored in the JavaSpace. Therefore, notification services can be implemented using JavaSpaces. However there are some problems with the function of event managers, so we implement JS-EM(JavaSpace-based Event Management System) by modifying JavaSpace. In this chapter we briefly introduce JavaSpaces and then review the issues when we implement event managers with pure JavaSpace. After that, we explain how we implement our event manager.

\subsection{JavaSpaces}

JavaSpaces[7] are a Jini service that allows clients to share objects. Its goal is to facilitate cooperative distributed computing. It is a reliable distributed storage system. JavaSpaces should be thought of as a "place" in which it is possible to store and share information in the form of objects. Objects flow around space-based systems, allowing clients to share and store information and also, via the use of dynamic class loading, share behavior. In JavaSpaces, all objects must also implement the Entry interface. Entries are found in association or matching.

JavaSpaces use templates to match entries within the Space by their field's values and type. JavaSpaces do use the value of the objects to do the matching. JavaSpaces export proxies to clients dynamically, via the Jini lookup service, so they are naturally distributed. JavaSpaces exist outside of other applications or services vecause they are themselves, but applications and services that use them import parts of the space to interact locally with the JavaSpaces interface.

\subsection{The Problems of Implementing Event Manager Using JavaSpaces}

Event managers can be implemented with JavaSpaces. Figure 1 shows the expected procedures of the event management when the event manager is implemented using JavaSpaces. There are, however, some issues with this implementation.

If more than one event producers write the same type of objects on JavaSpace and then event consumers are trying to take a object with a read() method, there is a problem that only firstly matched object will be returned. For example, let us assume that every 4 seconds, 4 events with the same type are written on JavaSpace, and their lease time is 10 seconds. In this case, as we can see in figure 2, from the second event object forth, there is the difference in event objects which event producers issued and event consumers read. 


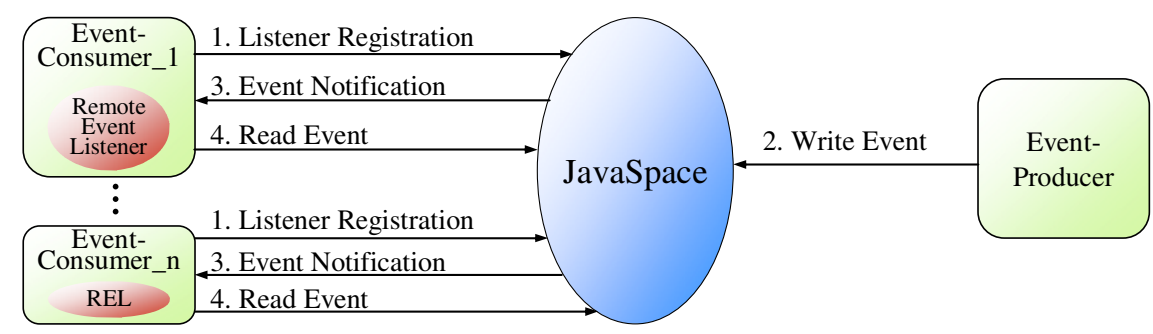

Fig. 1. Expected procedures of the event manager with JavaSpaces

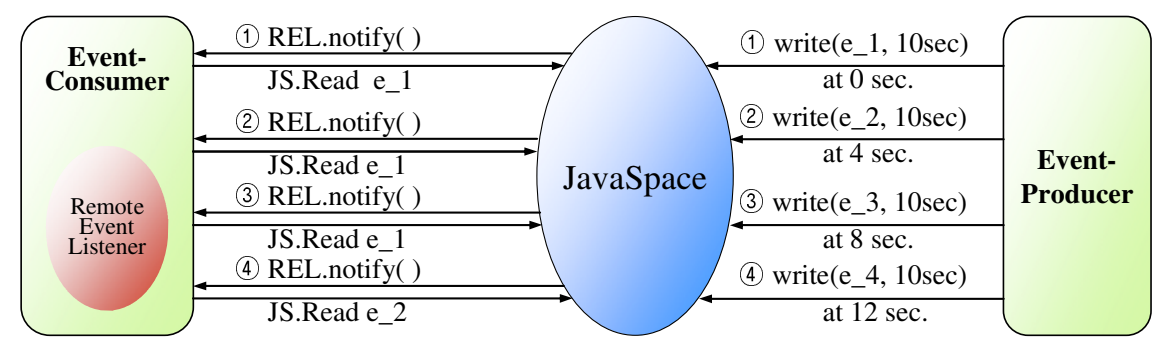

Fig. 2. Events taken by consumer with JavaSpace implementation

\subsection{JS-EM Using the Modified JavaSpace}

Our event manager (JS-EM : JavaSpace-based Event Manager) is made by modifying JavaSpace. In the existing JavaSpaces, event consumer should read the corresponding event by calling a read( ) method which is a JavaSpace API with the information of the event object which her event handler received. Granting that it can read events, there is no guarantee that it can obtain the correct one.

Hence by modifying JavaSpace for it to send the event object with the event notification as well, as in figure 3 , event consumers can immediately receive events without calling a read( ) method.

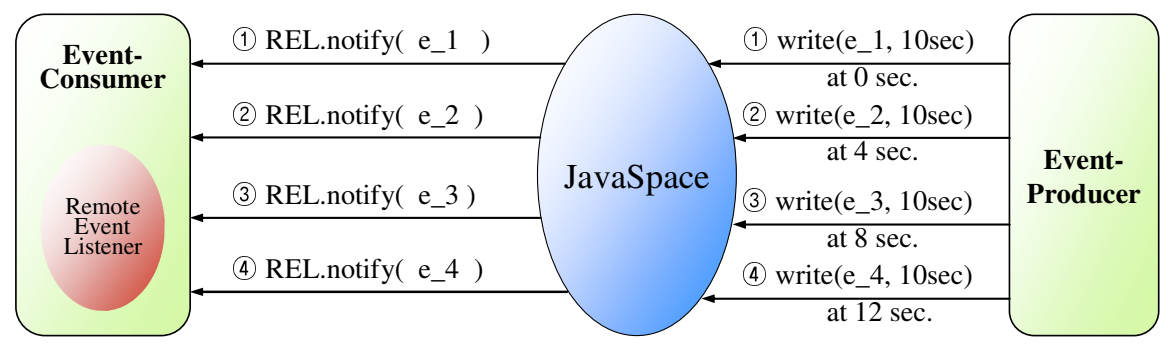

Fig. 3. Events consistency between sent events and obtained events

The procedure that the event consumer takes events generated by the event producer is as follows: 
1. As JS-EM itself is registered as service in the Jini LookUp service, the event consumers or the event producers can search the JS-EM and use it. An event consumer registers herself to JS-EM as a listener of the event he or she is interested in.

2. JS-EM takes the stub of event listener for the communication with the event consumer through web server and by doing this, there accomplishes the channel between event listener and JS-EM.

3. The event producers write its events to the JS-EM.

4. Events written to the channel in JS-EM are transmitted to the event consumer via previously registered event listener.

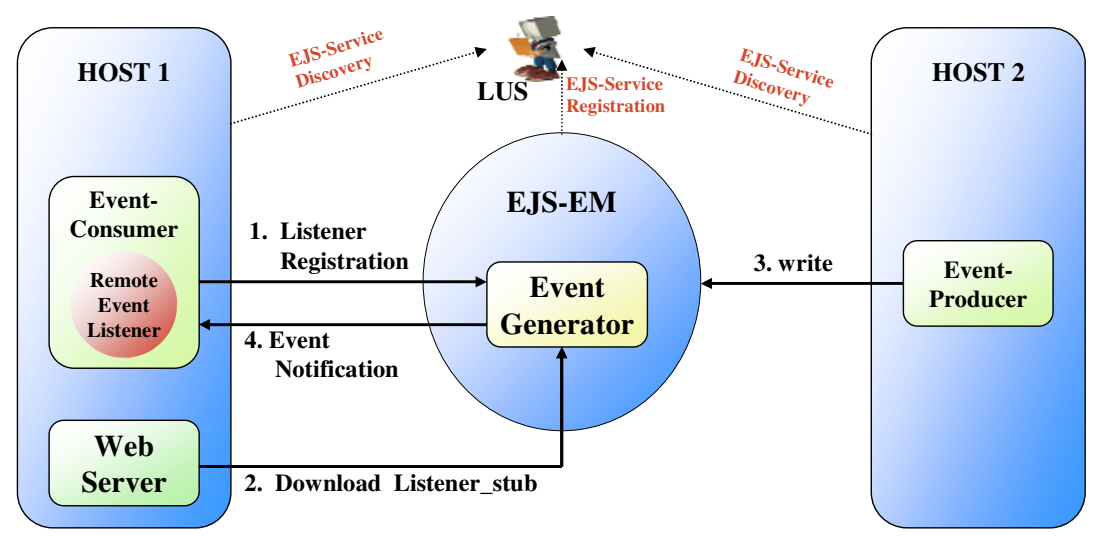

Fig. 4. Event service procedures using the extended JavaSpace

Our event manager provides a model for decoupled communication among event consumers and event producers. It allows creating and deleting channels. And through our event manager service, the context management service is able to generate the high level context information.

In a room, if a sensor sends location position information of a person after sensing, the consumer who tries to achieve the event is able to take the event through JS-EM. It means that the event is saved in the event channel generated by event manager, and then more than two event consumers, registered in the event channel as listener, are able to receive the event whenever the event producer writes the event into the event channel.

\section{Prototype Implementation and Analysis}

Our prototype is implemented in Linux/Windows OS, JDK 1.3 and Jini 1.2 development environment. We implemented our event manager by modifying JavaSpaces as described at chapter 3. In this chapter we describe how event managers having our proposed extended JavaSapce can be utilized. To implement our prototype first we 
propose architecture and then make scenarios. After that, we implement the application of each scenario by using Macromedia's flash and Sun's Java.

\subsection{Architecture}

In figure 5 which is the overall architecture of our prototype which we want to implement, it describes how application devices and sensor devices can use our event managers. TV itself, an application device, registers at JS-EM to listen to needed events. Sensor devices write their issued events on JS-EM, and JS-EM notifies it to a context manager.

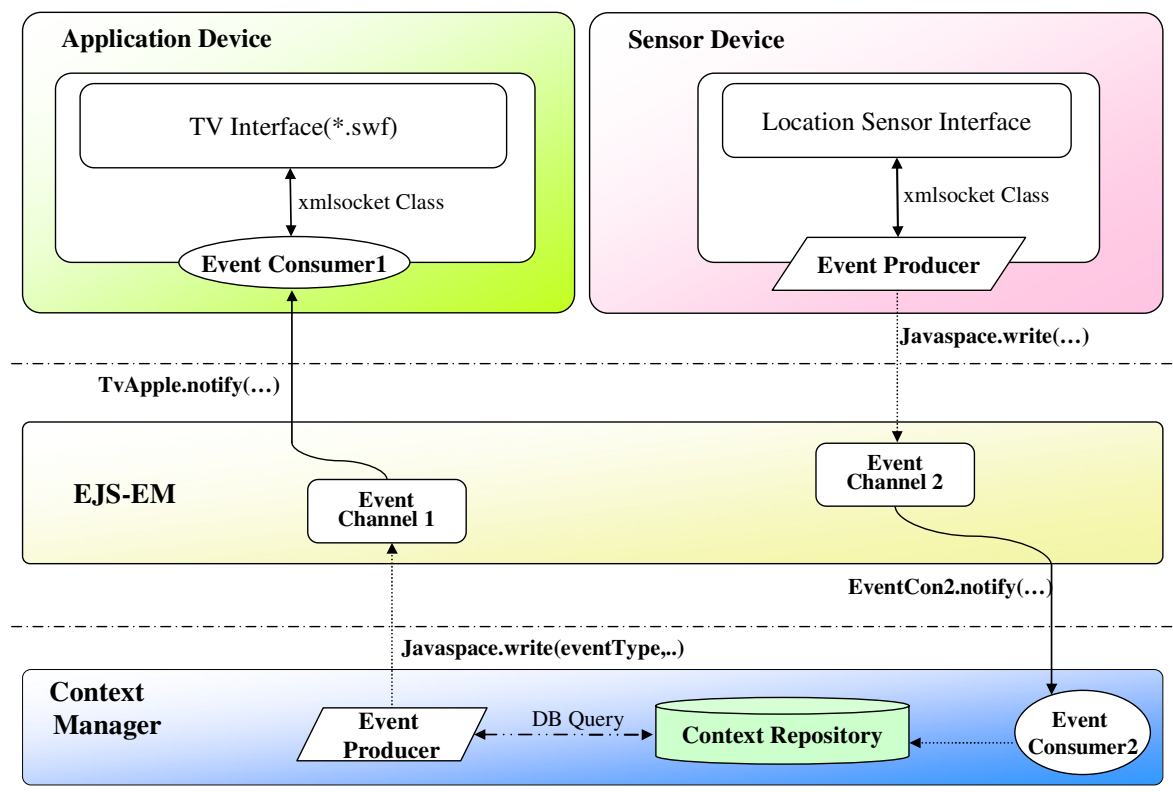

Fig. 5. Architecture of our event system

\subsubsection{Devices}

For our demonstration we made the virtual S/W devices with Macromedia's flash. Necessary devices for the demonstration are as follows.

1) Sensor devices: Transmit the event informations of location, temperature, and pressure to the context manager.

2) Application devices: Devices, such as a TV or a refrigerator, which adapts to events coming from context managers

\subsubsection{Event Manager}

Event managers play roles of conveying issued events from sensors to the context manager or transmitting high-quality events collected by the context manager to application layer's devices. 


\subsubsection{Context Manager}

The context manager processes context information coming from sensors, stores it, and converts it into high layer's context information according to the inference policy. This context information is the events which application layer's devices want, so to inference those events the context manager provides GUI for setting the inference policy. As this component is beyond our paper, we omit the explanation for this.

\subsection{Scenario}

The scenario for our prototype implementation is as follows.

6 PM, July 10, 2005. After finishing her daily work, Alice headed to home. On her way home she stopped by a fruit store. When she arrived at her home, the light of the porch automatically turned on, and she entered after authentication. First she changed her dress in her room, ate dinner, and sat down on the sofa. The context manager which recognized that Alice sat down on the sofa transmitted her favorite channel information to TV based on her preference and turned on TV. During watching TV, she recognized that she left her wallet in the fruit store. So she left her house in a hurry with TV on. TV stored the current TV status and turned off itself after receiving an event from the context manager representing Alice left. When she came back and sat on a sofa, the TV channel which she saw right before she left is on.

\subsection{TV On/Off Algorithm}

The on/off event classes which a TV device received is as follows.

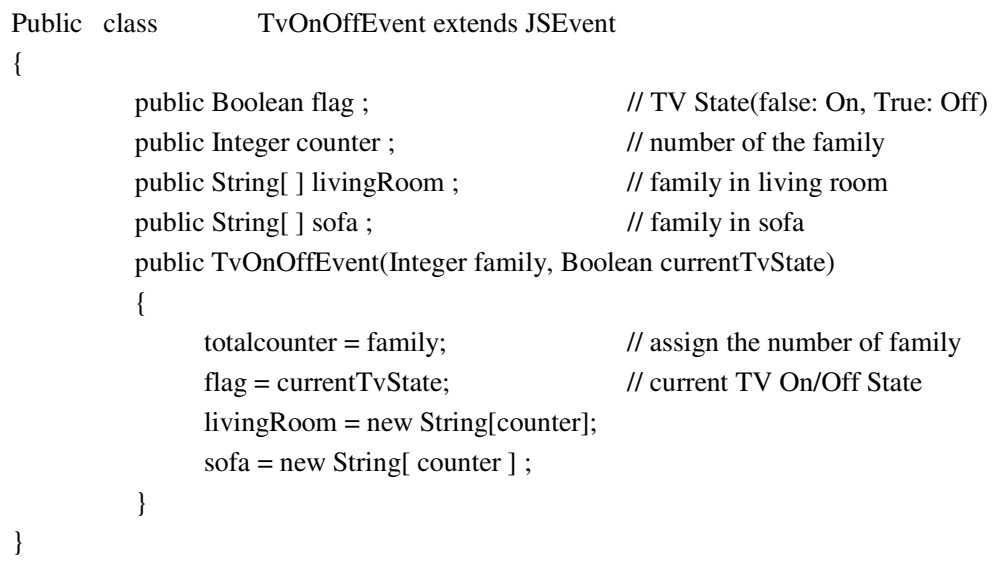

The adaptation algorithm of event consumer after receiving TV's on/off events is as follows. 


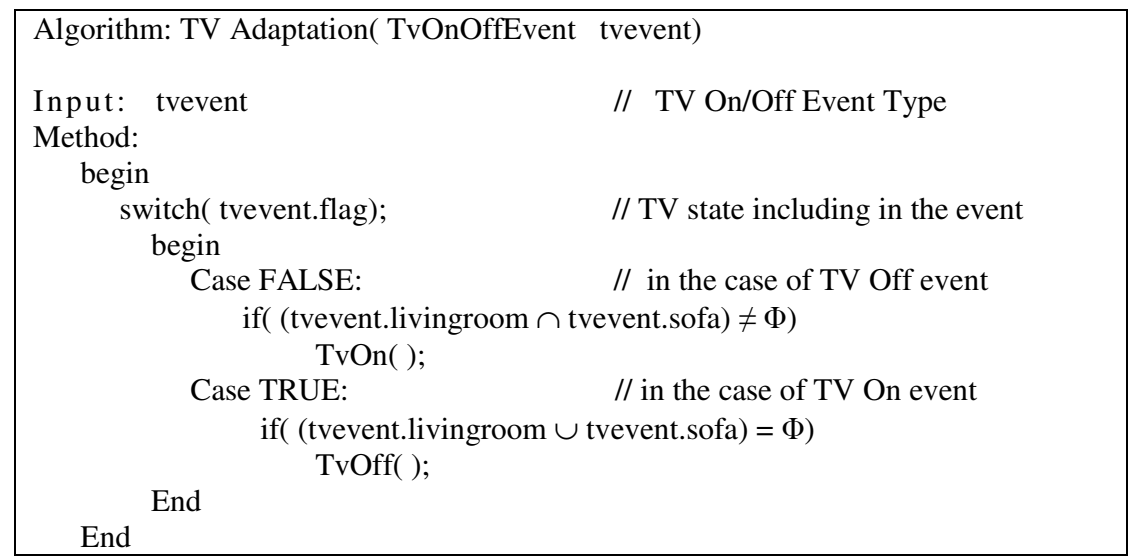

Fig. 6. TV On/Off Adaptation Algorithm

\subsection{TV On/Off Demonstration}

The procedures for TV to be on or off are as follows.

1. A TV application device registers listener at JS-EM to obtain on/off event.

2. If low level events are issued from sensors, the context manager infers the high level's events from them which can make TV on or off, then notifies a TV device via an event manager.

3. The TV device adapts to on/off events

Below figure 7 shows our prototype implementing the previous scenario.

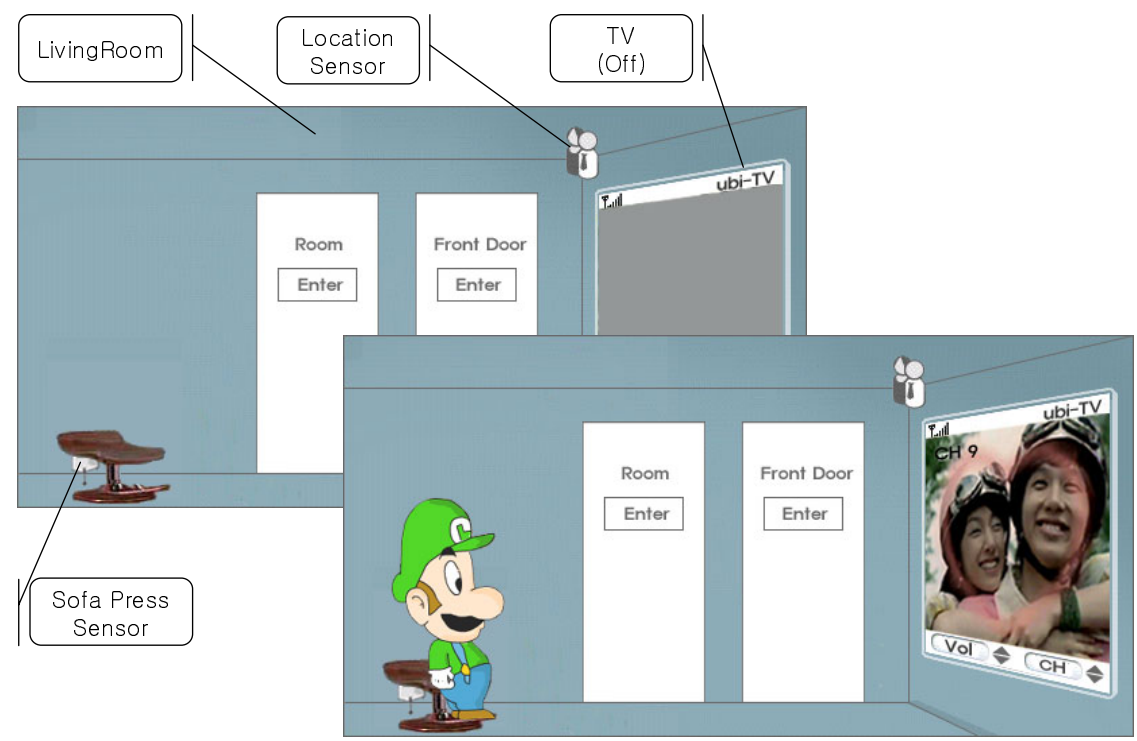

Fig. 7. Demonstration of our prototype implementing our scenario 


\subsection{System Analysis}

As our event manager, JS-EM, has whole advantages of JavaSpace, it is cooperative and scales well for loosely-coupled systems. In our JS-EM, if the event producer write events on JS-EM with a write( ) method, then it conveys an event object to the consumer through the parameter of a notify( ) method. When we compare JavaSpace with our JS-EM from the view of event management the result is like Table 1.

Table 1. The comparison of JavaSpaces and JS-EM

\begin{tabular}{|c|c|c|c|}
\hline & & JavaSpaces & Our JS-EM \\
\hline \multirow{6}{*}{$\begin{array}{c}\text { Num } \\
\text { of } \\
\text { call }\end{array}$} & Listener registration & 5 & 5 \\
\hline & write( event ) & 1 & 1 \\
\hline & Listener match module & 1 & 1 \\
\hline & notify( ) & 5 & 5 \\
\hline & $\operatorname{Read}($ ) & 5 & 0 \\
\hline & Object match module & 5 & 0 \\
\hline & Characteristics & $\begin{array}{l}\text { Event consumers } \\
\text { cannot make sure that } \\
\text { they are taking the } \\
\text { even notified by } \\
\text { JavaSpace. }\end{array}$ & $\begin{array}{l}\text { Event consumers } \\
\text { can make sure that } \\
\text { they are taking the } \\
\text { event notified by } \\
\text { JS-EM }\end{array}$ \\
\hline
\end{tabular}

In table 1, we assume that there are five clients waiting for one same type event. This table shows that our JavaSpace much faster and gives less load because it does not call read( ) and object match modules. In the previous JavaSpace, as it returns the firstly matched object when calling a read( ) method, there is no guarantee that the very event object which issued the event returns. On the other hand, in the modified JavaSpace, as it returns the object which issued the event when notifying the event consumer, the object which issued the event can be correctly transmitted.

\section{Conclusions and Further Work}

In the ubiquitous environments, above all adaptation is the most important. For this, we made the event manager so that we can manage events in distributed environments by modifying JavaSpace. As our event manager, JS-EM, inherits the properties of JavaSpace, it can have whole advantages of JavaSpace as it is.

In the future work, we want to control events so that only owner who has the right authority can take events by extending our Jini. We will clarify differences between context manager and event manager in our further research. Formalization of ACLs is important because it possibly moves with events. In addition, we should consider where to put ACL related to event authorization. In our prototype, we only put it in the event producer in order to observe its possibility. 
Because in order to minimize the event manager's burden, the event manager only relays the event, the trust checking on the side of the event consumer is in the right process, appropriate encoding is necessary. In a sense that computing power of the event consumer is available, the first or the third method is prominent in this paper. Within the ubiquitous environment, security entities should be assigned as new service, because client should keep working with substitute service.

\section{References}

1. CORBA services: Common Object Services Specification, OMG Specification, Object Management Group, Nov (1997)

2. Siegel, J.: CORBA 3: Fundamentals and Programming, Object Management Group (2000)

3. B.Borthakur: Distributed and Persistend Event System For Active Spaces. In: Master Thesis in Computer Science, Urbana-Champaign: University of Illinois at Urbana-Champaign (2002)

4. Richard Monson-Haefel, David Chappel: Java Message Service, O'Reilly (2000)

5. W.Keith Edwards, W.Edwards: Core Jini, Pearson Education (2000)

6. Rosanna Lee, Scott Seligman: JNDI API Tutorial and Reference: building directory-enabled $\mathrm{Java}^{\mathrm{TM}}$ Applications(Paperback), Addison-Wesley Professional (2000)

7. Philip Bishop and Nigel Warren: JavaSpaces IN PRACTICE, Addison-Wesley (2003) 